\title{
Fine\#Structure Excitation of Oiand Ciby Impact with Atomic Hydrogen
}

\section{Citation}

Abrahamsson, E., R. V. Krems, and A. Dalgarno. 2007. "Fine\#Structure Excitation of Oiand Ciby Impact with Atomic Hydrogen." The Astrophysical Journal 654 (2): 1171-74. https:// doi.org/10.1086/509631.

\section{Permanent link}

http://nrs.harvard.edu/urn-3:HUL.InstRepos:41397376

\section{Terms of Use}

This article was downloaded from Harvard University's DASH repository, and is made available under the terms and conditions applicable to Other Posted Material, as set forth at http:// nrs.harvard.edu/urn-3:HUL.InstRepos:dash.current.terms-of-use\#LAA

\section{Share Your Story}

The Harvard community has made this article openly available.

Please share how this access benefits you. Submit a story.

Accessibility 
The Astrophysical Journal, 654:1171-1174, 2007 January 10

(C) 2007. The American Astronomical Society. All rights reserved. Printed in U.S.A.

\title{
FINE-STRUCTURE EXCITATION OF O I AND C I BY IMPACT WITH ATOMIC HYDROGEN
}

\author{
E. Abrahamsson and R. V. Krems \\ Department of Chemistry, University of British Columbia, Vancouver, BC, Canada; rkrems@chem.ubc.ca
}

AND

A. Dalgarno

ITAMP, Harvard-Smithsonian Center for Astrophysics, Cambridge, MA

Received 2006 July 6; accepted 2006 September 20

\begin{abstract}
Using accurate interaction potentials, we perform refined calculations of rate coefficients for the fine-structure excitations in collisions of $\left.\mathrm{O}^{3} P\right)$ and $\mathrm{C}\left({ }^{3} P\right)$ with atomic hydrogen. The results are presented in the form of analytical functions approximating the rate coefficients over a wide range of temperatures. We examine the sensitivity of the collision dynamics to variations of the interaction potentials and the couplings to electronically excited states.
\end{abstract}

Subject headings: astrochemistry — atomic processes — methods: numerical — molecular processes — scattering

\section{INTRODUCTION}

The excitation of the fine-structure levels of $\mathrm{O}_{\mathrm{I}}$ and $\mathrm{C}_{\mathrm{I}}$ in collisions with hydrogen atoms contribute significantly to the cooling of diffuse interstellar gas (Dalgarno \& McCray 1972; Cowie \& Songaila 1986; Shaw et al. 2006). The intensities of the spectral lines that arise are also diagnostic probes of the environment of the atoms. The emission lines are strong in photon-dominated regions (PDRs; Kramer et al. 2004; Bernard-Salas \& Tielens 2005; Bensch 2006). They have been used as mass tracers of galaxies (Papandopoulos \& Greve 2004) and as indicators of the evolutionary stages of molecular clouds (Oka et al. 2004). The absorption line intensities provide a measure of the cosmic background temperature at high redshifts (Ge et al. 1997; Quast et al. 2002). The interpretation of the observational data depends on the rates of the excitation and deexcitation processes (Pequignot 1990).

The previous calculations of rate coefficients for fine-structure excitations in $\mathrm{O}-\mathrm{H}$ and $\mathrm{C}-\mathrm{H}$ collisions were reported by Launay \& Roueff (1977). Their results were based on model interaction potentials and a sparse energy grid of cross sections. The interaction potentials of the $\mathrm{OH}$ and $\mathrm{CH}$ molecules that determine the collision problem have been calculated, subsequently, with much greater precision (Yarkony 1992; Parlant \& Yarkony 1999; Kalemos et al. 1999) and a refined study of fine-structure excitation rates is warranted.

The interactions of $\mathrm{O}\left({ }^{3} P\right)$ and $\mathrm{C}\left({ }^{3} P\right)$ with hydrogen are determined by four molecular potentials of the ${ }^{2} \Sigma,{ }^{2} \Pi,{ }^{4} \Sigma$, and ${ }^{4} \Pi$ symmetries. These potentials have been recently computed by Parlant \& Yarkony (1999) for the OH molecule and Kalemos et al. (1999) for the CH molecule. In this work, we use the results of Parlant \& Yarkony and Kalemos et al. to improve on the calculations of Launay \& Roeuff (1977). We explore the sensitivity of the rate coefficients to the interaction potentials and present analytical formulae for the temperature dependence of the rate coefficients. The energy of the first electronic excitation of $\mathrm{O}\left({ }^{3} P\right)$ to the ${ }^{1} D$ state is about $2 \mathrm{eV}$, and the ${ }^{1} D$ state is usually ignored in calculations of collision dynamics involving $\mathrm{O}\left({ }^{3} P\right)$ at low temperatures. The interaction of $\mathrm{O}\left({ }^{1} D\right)$ with hydrogen is, however, characterized by strongly attractive interaction potentials that are coupled to the ${ }^{2} \Sigma$, ${ }^{2} \Pi,{ }^{4} \Sigma$, and ${ }^{4} \Pi$ states of the $\mathrm{OH}$ molecule by the spin-orbit interaction (Krems et al. 2006) and may modify the dynamics of $\mathrm{O}\left({ }^{3} P\right)-\mathrm{H}$ colli- sions at high temperatures. We show that these couplings are insignificant.

\section{CALCULATION DETAILS}

We calculate the cross sections for $\mathrm{C}-\mathrm{H}$ and $\mathrm{O}-\mathrm{H}$ collisions using the time-independent quantum mechanical approach as described by Launay (1977). The spin-orbit interaction splits the ground electronic state of oxygen and carbon into a triplet of fine-structure levels ${ }^{3} P_{0},{ }^{3} P_{1}$, and ${ }^{3} P_{2}$. The lowest energy level of oxygen is ${ }^{3} P_{2}$ and that of carbon is ${ }^{3} P_{0}$. Following the National Institute of Standards and Technology (NIST) database, ${ }^{1}$ we adopt the energies $158 \mathrm{~cm}^{-1}$ for the ${ }^{3} P_{1}$ state and $227 \mathrm{~cm}^{-1}$ for the ${ }^{3} P_{0}$ state, relative to the ${ }^{3} P_{2}$ state for oxygen. For carbon, we use the energies $16.4 \mathrm{~cm}^{-1}$ for the ${ }^{3} P_{1}$ state and $43.4 \mathrm{~cm}^{-1}$ for the ${ }^{3} P_{2}$ state, relative to the ${ }^{3} P_{0}$ state. Within the closecoupling method (Arthurs \& Dalgarno 1960) the scattering problem is reduced to a set of coupled second-order differential equations parameterized by the total energy and the total angular momentum of the collision complex. We solve these equations on a grid of 1450 kinetic energies in the interval from 1 to $10,000 \mathrm{~cm}^{-1}$ and the total angular momenta $J=0-100$. The solutions yield the scattering $S$-matrices and the cross sections for the fine-structure transitions (Launay 1977). The rate coefficients are calculated by averaging the product of the cross sections and the relative velocity over a Maxwellian velocity distribution. To generate the interaction potentials from the data of Parlant \& Yarkony (1999), we used spline interpolation smoothly joined with the long-range analytical form of the interatomic interaction. The interaction potential data of Kalemos et al. (1999) were interpolated and extrapolated using the method described by Ho \& Rabitz (1996).

\section{RESULTS}

Table 1 lists the rate coefficients for the fine-structure excitations in $\mathrm{C}\left({ }^{3} P\right)-\mathrm{H}$ collisions. The results in the "Accurate" columns are obtained using accurate interaction potentials of Kalemos et al. (1999). To estimate the uncertainties of our calculations and explore the sensitivity of the rate coefficients to the molecular potentials, we modified the short-range part of

\footnotetext{
${ }^{1}$ See http://physics.nist.gov/PhysRef Data/Handbook/index.html.
} 
TABLE 1

Rate Coefficients for the Fine-Structure Excitations in $\mathrm{C}\left({ }^{3} P\right)+\mathrm{H}$ Collisions

\begin{tabular}{|c|c|c|c|c|c|c|c|c|c|}
\hline \multirow{2}{*}{$\begin{array}{c}T \\
(\mathrm{~K})\end{array}$} & \multicolumn{3}{|c|}{$\begin{array}{c}k_{0 \rightarrow 1} \\
\left(10^{-11} \mathrm{~cm}^{3} \mathrm{~s}^{-1}\right)\end{array}$} & \multicolumn{3}{|c|}{$\begin{array}{c}k_{0 \rightarrow 2} \\
\left(10^{-11} \mathrm{~cm}^{3} \mathrm{~s}^{-1}\right)\end{array}$} & \multicolumn{3}{|c|}{$\begin{array}{c}k_{1 \rightarrow 2} \\
\left(10^{-11} \mathrm{~cm}^{3} \mathrm{~s}^{-1}\right)\end{array}$} \\
\hline & $\mathrm{LR}_{7} 7^{\mathrm{a}}$ & Accurate $^{\mathrm{b}}$ & Modified $^{c}$ & $\mathrm{LR} 77^{\mathrm{a}}$ & Accurate $^{\mathrm{b}}$ & Modified $^{c}$ & $\mathrm{LR} 77^{\mathrm{a}}$ & Accurate $^{\mathrm{b}}$ & Modified $^{\mathrm{c}}$ \\
\hline$\ldots \ldots$. & 0.13 & 0.122 & 0.222 & $\ldots$ & $\ldots$ & 0.0191 & $\ldots$ & 0.0021 & 0.0340 \\
\hline $5 \ldots \ldots$ & 0.42 & 0.382 & 0.609 & $\ldots$ & 0.0001 & 0.0239 & $\ldots$ & 0.0146 & 0.0508 \\
\hline ....................... & 1.64 & 1.40 & 2.16 & $\ldots$ & 0.0046 & 0.0367 & 0.15 & 0.132 & 0.190 \\
\hline $8 \ldots \ldots \ldots \ldots \ldots . .$. & 2.51 & 2.10 & 3.23 & $\ldots$ & 0.0139 & 0.0491 & 0.30 & 0.261 & 0.345 \\
\hline ................... & 3.51 & 2.87 & 4.42 & $\ldots$ & 0.0327 & 0.0701 & 0.52 & 0.444 & 0.568 \\
\hline $10 \ldots \ldots \ldots$ & 4.59 & 3.68 & 5.67 & 0.10 & 0.065 & 0.103 & 0.80 & 0.678 & 0.857 \\
\hline $12 \ldots \ldots \ldots$ & 6.87 & 5.33 & 8.23 & 0.27 & 0.180 & 0.218 & 1.54 & 1.28 & 1.61 \\
\hline $14 \ldots \ldots \ldots \ldots \ldots$ & 9.17 & 6.93 & 10.70 & 0.57 & 0.3724 & 0.4084 & 2.45 & 2.02 & 2.55 \\
\hline 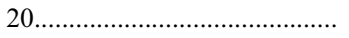 & 15.20 & 11.08 & 16.95 & 2.14 & 1.39 & 1.43 & 5.76 & 4.61 & 5.90 \\
\hline 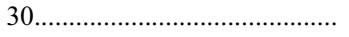 & 22.20 & 16.00 & 23.87 & 6.00 & 4.00 & 4.14 & 11.40 & 9.03 & 11.52 \\
\hline 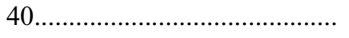 & 26.50 & 19.44 & 28.18 & 10.00 & 7.035 & 7.343 & 16.20 & 13.02 & 16.37 \\
\hline 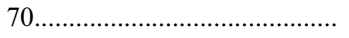 & 33.80 & 25.86 & 35.16 & 19.20 & 16.08 & 16.86 & 26.20 & 22.59 & 27.04 \\
\hline $100 \ldots \ldots \ldots \ldots$ & 38.00 & 29.75 & 38.97 & 25.10 & 23.83 & 24.90 & 32.40 & 29.79 & 34.43 \\
\hline 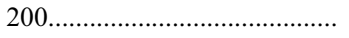 & 45.20 & 37.56 & 46.38 & 37.40 & 41.19 & 42.71 & 44.50 & 45.20 & 49.71 \\
\hline 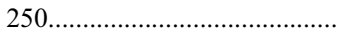 & 47.50 & 40.41 & 49.12 & 42.10 & 46.94 & 48.62 & 48.70 & 50.41 & 54.92 \\
\hline (n...................... & 48.90 & 42.90 & 51.53 & 46.20 & 51.70 & 53.51 & 52.40 & 54.79 & 59.33 \\
\hline 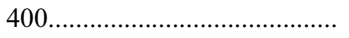 & 51.80 & 47.12 & 55.68 & 53.20 & 59.45 & 61.46 & 58.80 & 62.06 & 66.70 \\
\hline 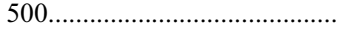 & 54.60 & 50.68 & 59.20 & 59.10 & 65.80 & 67.94 & 64.30 & 68.12 & 72.86 \\
\hline (n) & 59.60 & 56.67 & 65.12 & 68.80 & 76.20 & 78.46 & 73.70 & 78.24 & 83.07 \\
\hline 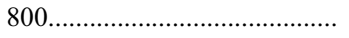 & 62.00 & 59.32 & 67.70 & 73.00 & 80.67 & 82.92 & 77.80 & 82.65 & 87.48 \\
\hline $900 \ldots \ldots \ldots \ldots \ldots \ldots \ldots \ldots . .$. & 64.20 & 61.80 & 70.08 & 76.90 & 84.80 & 87.00 & 81.70 & 86.75 & 91.52 \\
\hline $1000 \ldots \ldots \ldots \ldots \ldots \ldots \ldots$ & 66.30 & 64.15 & 72.28 & 80.60 & 88.64 & 90.75 & 85.30 & 90.61 & 95.26 \\
\hline
\end{tabular}

a Data of Launay \& Roueff (1977).

b This work: accurate results.

c This work: calculations with modified potentials.
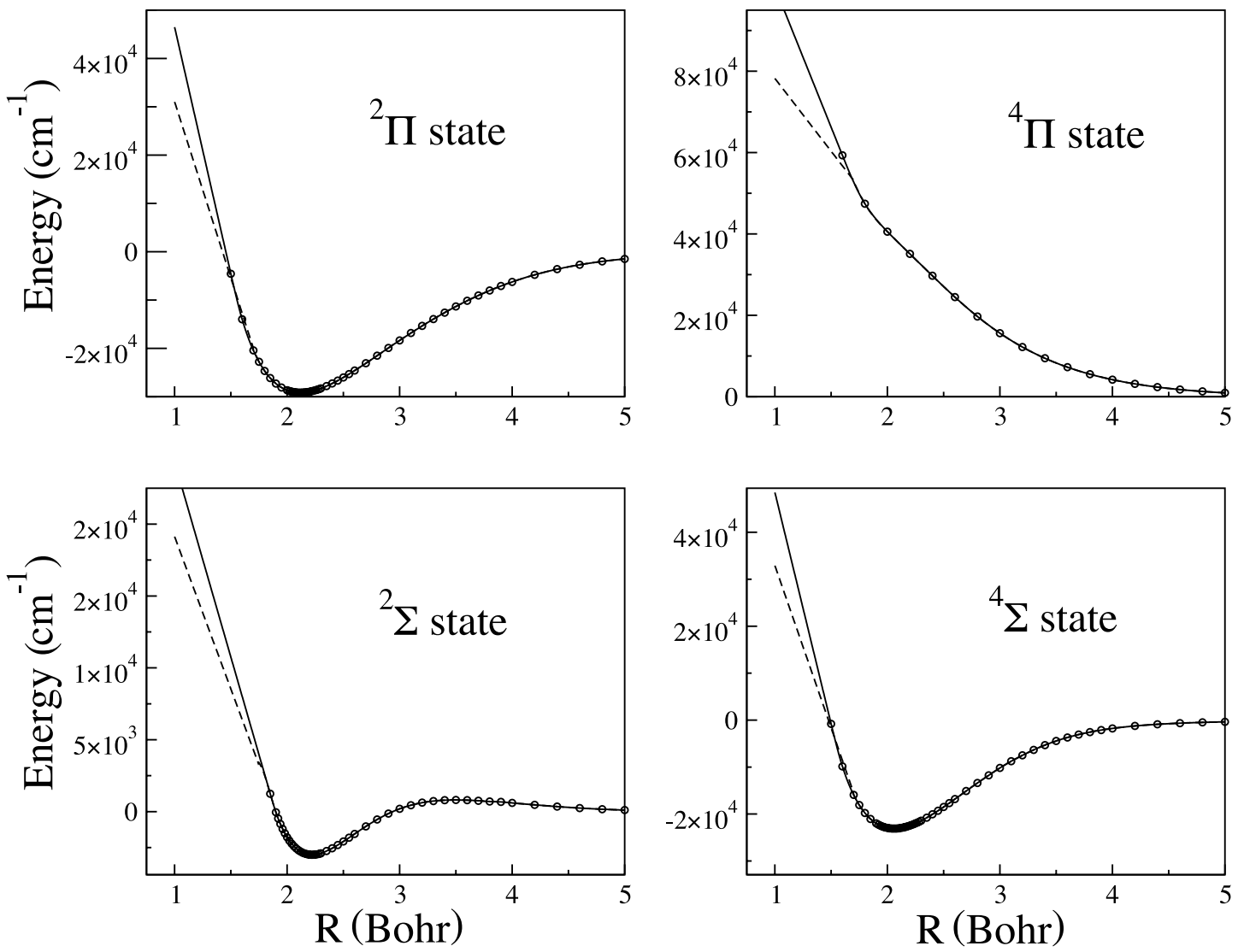

Fig. 1. - Interaction potentials of the $\mathrm{C}\left({ }^{3} P\right) \mathrm{H}$ molecule. Circles: ab initio calculations of Kalemos et al. (1999); solid curves: numerical interpolation of the ab initio data as described by Ho \& Rabitz (1996); dashed curves: potentials with modified short-range interactions. 
TABLE 2

Fitting Coefficients to Represent the Rate Coefficients for the Fine-Structure Excitations in $\mathrm{C}+\mathrm{H}$ Cololisions by eQ. (1)

\begin{tabular}{|c|c|c|c|}
\hline Excitation & $j=0 \rightarrow j^{\prime}=1$ & $j=0 \rightarrow j^{\prime}=2$ & $j=1 \rightarrow j^{\prime}=2$ \\
\hline & 3.6593 & 10.8377 & 15.8996 \\
\hline & 56.6023 & -173.4153 & -201.3030 \\
\hline $\overrightarrow{2}$ & -802.9765 & 2024.0272 & 1533.6164 \\
\hline ......... & 5025.1882 & -13391.6549 & -6491.0083 \\
\hline & -17874.4255 & 52198.5522 & 15921.9239 \\
\hline$J_{(5)}^{J}$ & 38343.6655 & -124518.3586 & -22691.1632 \\
\hline$a_{j \rightarrow j^{\prime}}^{(\vec{b})^{\prime}}$ & -49249.4895 & 178182.5823 & 17334.7529 \\
\hline & 34789.3941 & -140970.6106 & -5517.9360 \\
\hline$a_{j \rightarrow j^{\prime}}^{(8)}$ & -10390.9809 & 47504.5861 & 0 \\
\hline$b$ & $1 / 4$ & $1 / 3$ & $1 / 4$ \\
\hline
\end{tabular}

the interaction potentials as shown in Figure 1. The dashed curves of Figure 1 are smoothly joined with the ab initio data at the interatomic distances $R>1.7$ bohr. The "Modified" columns of Table 1 list the rate coefficients calculated with the modified potentials. Our results differ from the previous calculations of Launay \& Roueff (1977) by up to $20 \%$ at low temperatures and agree well with their data at temperatures above $700 \mathrm{~K}$. Given the model nature of the interaction potentials used by Launay \& Roueff (1977) and the sensitivity of the dynamics to the interaction potentials demonstrated by Table 1, we find the agreement remarkable and coincidental.

At temperatures between 5 and $1000 \mathrm{~K}$, the rate coefficients for the fine-structure excitations are accurately represented by the formula

$$
\ln \left(\frac{k_{j \rightarrow j^{\prime}}}{10^{-11}}\right)=\sum_{i=0}^{N} a_{j \rightarrow j^{\prime}}^{(i)} T^{-i b},
$$

with the fitting coefficients listed in Table 2 .

Table 3 presents the rate coefficients for the fine-structure excitations in $\mathrm{O}\left({ }^{3} P\right)-\mathrm{H}$ collisions. The calculations for oxygen are based on accurate interaction potentials computed and presented by Parlant \& Yarkony (1999). The previous calculations
TABLE 4

Fitting Coefficients to Represent the Rate Coefficients for the Fine-Structure Excitations in $\mathrm{O}+\mathrm{H}$ Collisions by eQ. (1)

\begin{tabular}{|c|c|c|c|}
\hline Excitation & $j=2 \rightarrow j^{\prime}=1$ & $j=2 \rightarrow j^{\prime}=0$ & $j=1 \rightarrow j^{\prime}=0$ \\
\hline & 4.581 & 3.297 & 3.437 \\
\hline$a_{j \rightarrow j^{\prime}}^{(1)^{\prime}}$ & -156.118 & -168.382 & 17.443 \\
\hline$a_{j \rightarrow j^{\prime}}^{(2)^{\prime}}$ & 2679.979 & 1844.099 & -618.761 \\
\hline$a_{j \rightarrow j^{\prime}}^{(3)^{j}}$ & -78996.962 & -68362.889 & 3757.156 \\
\hline$a_{j \rightarrow j^{\prime}}^{(4)^{\prime}}$. & 1308323.468 & 1376864.737 & -12736.468 \\
\hline$a_{j \rightarrow j^{\prime}}^{(5)^{\prime}}$. & -13011761.861 & -17964610.169 & 22785.266 \\
\hline$a_{j \rightarrow j^{\prime}}^{(6)} \ldots \ldots \ldots \ldots \ldots \ldots$ & 71010784.971 & 134374927.808 & -22759.228 \\
\hline$a_{j \rightarrow j^{\prime}}^{(7)^{\prime}} \ldots \ldots \ldots \ldots \ldots \ldots$ & -162826621.855 & -430107587.886 & 12668.261 \\
\hline$b$ & $3 / 4$ & $3 / 4$ & $1 / 2$ \\
\hline
\end{tabular}

of Launay \& Roueff (1977) underestimate our more accurate results by up to a factor of 4 at low temperatures and a factor of 2-3 at temperatures near $1000 \mathrm{~K}$. At temperatures between 30 and $1000 \mathrm{~K}$, the rate coefficients of Table 3 are accurately represented by equation (1) with the fitting coefficients listed in Table 4. For temperatures below $30 \mathrm{~K}$ the rate coefficients are less than $10^{-14} \mathrm{~cm}^{3} \mathrm{~s}^{-1}$.

The energy of the first electronic excitation of $\mathrm{O}\left({ }^{3} P\right)$ to the ${ }^{1} D$ state is about $2 \mathrm{eV}$ and the calculations of the fine-structure excitation rates reported here and previously (Launay \& Roueff 1977) were performed with the basis set neglecting the electronic excited state of oxygen. The interaction of $\mathrm{O}\left({ }^{1} D\right)$ with $\mathrm{H}$ is described by three interaction potentials: $A^{2} \Sigma^{+},{ }^{2} \Pi$, and ${ }^{2} \Delta$ (Krems et al. 2006). The $A^{2} \Sigma^{+}$potential is strongly attractive. The spin-orbit and Coriolis interactions couple the $A^{2} \Sigma^{+}$ state with the molecular states ${ }^{2} \Sigma,{ }^{2} \Pi,{ }^{4} \Sigma$, and ${ }^{4} \Pi$ that determine the collision dynamics of $\mathrm{O}\left({ }^{3} P\right)$. The ${ }^{2} \Sigma,{ }^{2} \Pi,{ }^{4} \Sigma$, and ${ }^{4} \Pi$ states cross with the $A^{2} \Sigma^{+}$state at energies between 4000 and $10,000 \mathrm{~cm}^{-1}$, and the couplings to the ${ }^{1} D$ state may modify the dynamics of $\mathrm{O}\left({ }^{3} P\right)-\mathrm{H}$ collisions. To verify the accuracy of our calculations and explore the role of the couplings to the ${ }^{1} D$ state, we have written an independent computer program as described by Krems et al. (2006). Figure 2 compares the results of the extended calculations including the ${ }^{1} D$ state with the cross sections

TABLE 3

Rate Coefficients for the Fine-Structure Excitations in O $\left({ }^{3} P\right)+\mathrm{H}$ Collisions

\begin{tabular}{|c|c|c|c|c|c|c|}
\hline \multirow{2}{*}{$\begin{array}{c}T \\
(\mathrm{~K})\end{array}$} & \multicolumn{2}{|c|}{$\begin{array}{c}k_{2 \rightarrow 1} \\
\left(10^{-11} \mathrm{~cm}^{3} \mathrm{~s}^{-1}\right)\end{array}$} & \multicolumn{2}{|c|}{$\begin{array}{c}k_{2 \rightarrow 0} \\
\left(10^{-11} \mathrm{~cm}^{3} \mathrm{~s}^{-1}\right)\end{array}$} & \multicolumn{2}{|c|}{$\begin{array}{c}k_{1 \rightarrow 0} \\
\left(10^{-11} \mathrm{~cm}^{3} \mathrm{~s}^{-1}\right)\end{array}$} \\
\hline & $\mathrm{LR} 77^{\mathrm{a}}$ & This Work & $\mathrm{LR} 77^{\mathrm{a}}$ & This Work & $\mathrm{LR} 77^{\mathrm{a}}$ & This Work \\
\hline $50 \ldots \ldots \ldots \ldots$ & $\ldots$ & 0.189 & $\ldots$ & 0.0080 & 0.39 & 1.10 \\
\hline $70 \ldots \ldots \ldots \ldots$ & $\ldots$ & 0.740 & $\ldots$ & 0.0548 & $\ldots$ & 2.70 \\
\hline $100 \ldots \ldots$ & 0.56 & 2.20 & $\ldots$ & 0.243 & 1.33 & 5.38 \\
\hline $150 \ldots \ldots \ldots \ldots$ & 1.66 & 5.49 & 0.13 & 0.827 & 2.13 & 9.89 \\
\hline $200 \ldots \ldots \ldots \ldots$ & 2.98 & 9.06 & 0.29 & 1.59 & 2.81 & 13.80 \\
\hline . & 4.37 & 12.54 & 0.49 & 2.42 & 3.39 & 17.02 \\
\hline 300 & 5.74 & 15.83 & 0.71 & 3.24 & 3.92 & 19.65 \\
\hline 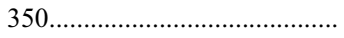 & 7.06 & 18.90 & 0.94 & 4.04 & 4.40 & 21.79 \\
\hline . & 8.33 & 21.74 & 1.18 & 4.80 & 4.85 & 23.55 \\
\hline $450 \ldots \ldots \ldots \ldots$. & 9.54 & 24.36 & 1.42 & 5.51 & 5.28 & 25.00 \\
\hline 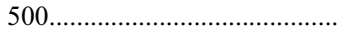 & 10.69 & 26.79 & 1.66 & 6.18 & 5.69 & 26.21 \\
\hline 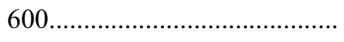 & 12.80 & 31.14 & 2.13 & 7.40 & 6.48 & 28.11 \\
\hline 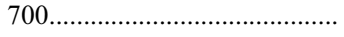 & 14.80 & 34.91 & 2.57 & 8.47 & 7.21 & 29.49 \\
\hline 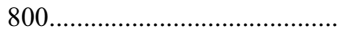 & 16.60 & 38.22 & 3.00 & 9.41 & 7.91 & 30.55 \\
\hline 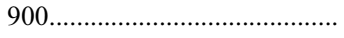 & 18.30 & 41.14 & 3.41 & 10.25 & 8.58 & 31.37 \\
\hline 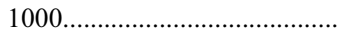 & 19.90 & 43.75 & 3.80 & 11.01 & 9.22 & 32.03 \\
\hline
\end{tabular}

a Data of Launay \& Roueff (1977). 


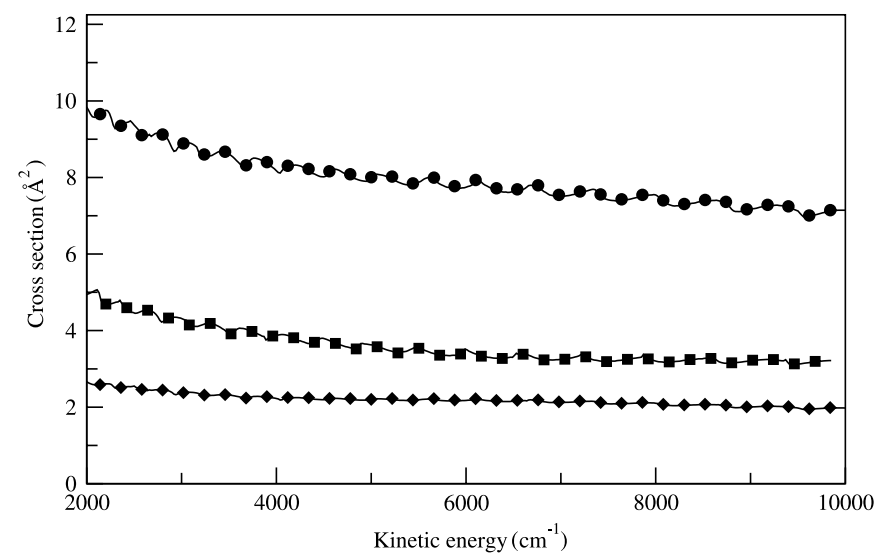

FIG. 2.-Cross sections for the fine-structure excitations in $\mathrm{O}\left({ }^{3} P\right)-\mathrm{H}$ collisions. Circles: $j=2 \rightarrow j=1$ transition; squares: $j=1 \rightarrow j=0$ transition; diamonds: $j=2 \rightarrow j=0$ transition. Solid curves represent calculations including the ${ }^{1} D$ state of oxygen as described by Krems et al. (2006).

computed using the conventional basis set of only the ${ }^{3} P$ states. The couplings to the ${ }^{1} D$ state appear to be insignificant even at high collision energies of $10,000 \mathrm{~cm}^{-1}$.

\section{SUMMARY}

Using accurate interaction potentials of Parlant \& Yarkony (1999) and Kalemos et al. (1999), we have recalculated the rate coefficients for the fine-structure excitations in collisions of $\mathrm{O}\left({ }^{3} P\right)$ and $\mathrm{C}\left({ }^{3} P\right)$ with atomic hydrogen. The results are presented in the form of analytical functions representing the rate coefficients over a wide range of temperatures. To verify the accuracy of the calculations, we examined the sensitivity of the rate coefficients to variations of the interaction potentials at short range and the couplings to electronically excited states of oxygen. This is the first calculation of fine-structure excitation dynamics of $\mathrm{O}\left({ }^{3} P\right)$ with the couplings to the ${ }^{1} D$ states included. Given the recent progress in interstellar spectroscopy measurements, the refined results presented here may improve our understanding of the conditions in the interstellar medium.

We thank Zhiying Li for verifying some of our results and for pointing out several misprints. We thank A. Kalemos and D. R. Yarkony for sending us the results of their potential energy calculations, and G. Barinovs for help with interpolating the interaction potentials for $\mathrm{CH}$. The work of E. A. and R. K. was supported by NASA grant NNG 06-GJ11G from the Astrophysics Theory Program and the Natural Sciences and Engineering Research Council (NSERC) of Canada and of A. D. by the Chemical Sciences, Geosciences and Biosciences Division of the Office of Basic Energy Sciences, Office of Science, US Department of Energy.
Arthurs, A. M., \& Dalgarno, A. 1960, Proc. R. Soc. London, Ser. A, 256, 540 Bensch, F. 2006, A\&A, 448, 1043

Bernard-Salas, J., \& Tielens, A. G. G. M. 2005, A\&A, 431, 523

Cowie, L. L., \& Songaila, A. 1986, ARA\&A, 24, 499

Dalgarno, A., \& McCray, R. A. 1972, ARA\&A, 10, 375

Ge, J., Bechtold, J., \& Black, J. H. 1997, ApJ, 474, 67

Ho, T.-S., \& Rabitz, H. 1996, J. Chem. Phys., 104, 2584

Kalemos, A., Mavridis, A., \& Metropoulos, A. 1999, J. Chem. Phys., 111, 9536

Kramer, C., Jakob, H., Mookerjea, B., Schneider, N., Brull, M., \& Stutzki, J. 2004, A\&A, 424, 887

Krems, R. V., Jamieson, M. J., \& Dalgarno, A. 2006, ApJ, 647, 1531

\section{REFERENCES}

Launay, J. M. 1977, J. Phys. B, 10, 3665

Launay, J. M., \& Roueff, E. 1977, A\&A, 56, 289

Oka, T., Iwata, M., Maezawa, H., Ikeda, M., Ito, T., Kamegai, K., Sakai, T., \& Yamamoto, S. 2004, ApJ, 602, 803

Papadopoulos, P. P., \& Greve, T. R. 2004, ApJ, 615, L29

Parlant, G., \& Yarkony, D. R. 1999, J. Chem. Phys., 110, 363

Pequignot, D. 1990, A\&A, 231, 499

Quast, R., Baade, R., \& Reimers, D. 2002, A\&A, 386, 796

Shaw, G., Ferland, G. J., Srianand, R., \& Abel, N. P. 2006, ApJ, 639, 941

Yarkony, D. R. 1992, J. Chem. Phys., 97, 1838 